\title{
A proposed model for understanding human-bacterial interactions: Space-time approach on community Escherichia coli occurrence and resistance phenomenon ${ }^{*}$
}

\author{
Carlos R. V. Kiffer ${ }^{1,2 \#}$, Antonio M. V. Monteiro ${ }^{3}$, Eduardo C. G. Camargo ${ }^{3}$, Antonio C. C. Pignatari ${ }^{1}$ \\ ${ }^{1}$ Laboratório Especial de Microbiologia Clinica, Department Doenças Infecciosas e Parasitárias, Universidade Federal de São Paulo, \\ São Paulo, Brazil \\ ${ }^{2}$ GC-2 Lab Ltd., São Paulo, Brazil \\ ${ }^{3}$ Divisão de Processamento de Imagens, Instituto Nacional de Pesquisas Espaciais, São José dos Campos, Brazil \\ Email: "kiffer@uninet.com.br
}

Received 15 January 2013; revised 25 February 2013; accepted 30 March 2013

Copyright (c) 2013 Carlos R. V. Kiffer et al. This is an open access article distributed under the Creative Commons Attribution License, which permits unrestricted use, distribution, and reproduction in any medium, provided the original work is properly cited.

\begin{abstract}
Due to ecological effect, it is expected that population exposures to antimicrobial drugs may lead to microorganisms' modifications, occasionally leading to resistance emergence. The present review was based on previous empirical data and on related literature search for quantitative empirical models exploring the human-bacterial interactions. Our previous studies have shown the emergence of ciprofloxacin resistant (CIPR) Escherichia coli significantly related to previous specific levels of ciprofloxacin consumption and to urban clusters of CIP-R E. coli. The evidence of significant spatial clustering of antimicrobial resistance (ciprofloxacin resistance $E$. coli) reinforces the ecological effect hypothesis as a major drive in resistance emergence. In other words, human populations submitted to a certain ciprofloxacin or quinolone usage level may affect neighbours within certain geographical areas, not necessarily due to individual antimicrobial intake, but as a driving pressure over a modified circulating $E$. coli population. Apparently quantitative spatial-temporal analytical frameworks may be better for understanding human-bacterial interactions based on any of their epiphenomena (antimicrobial consumption, antimicrobial resistance, geno/phenotypic characteristics).
\end{abstract}

\footnotetext{
"Author Contributions: C.R.V.K. designed, collected, performed part of the analysis (Kernel), reviewed the data, and wrote the report. E.C.G.C. collected, standardized the database, critically reviewed the experiment design, and performed part of the analysis (Kernel, Voronnoi, Theme Maps, Model). A.C.C.P. critically reviewed the experiment and helped interpreting and discussing the microbiological data. A.M.V.M. coordinated, designed, supervised collection of data, reviewed the data, performed part of the analysis, and wrote the report.

"Corresponding author.
}

Keywords: Escherichia coli; Antimicrobial Agents; Microbiome

\section{INTRODUCTION}

Although obvious, it is important to reinforce that microorganisms exist irrespective of their pathogenicity potential. Actually, most of their existence in nature is not harmful to most species, yet being the opposite. However, as part of the complex relationship between man and microbes, human infections or colonisations are indeed one of many possible epiphenomena of microorganisms' existence [1], frequently due to an imbalance in the human-microbe interaction.

As part of this imbalance, mortality due to infections represents approximately $85 \%$ of all deaths worldwide, and community acquired ones are highly prevalent $[2,3]$. Furthermore, a considerable amount of infections or colonisations-related microorganisms present a risk of resistance acquisition to one or more antimicrobial agents [2,4,5]. As stated by Lipsitch and Samore [6], "the use of antimicrobial agents is a powerful selective force that promotes the emergence of resistant strains". In other words, it is well established that the use of antimicrobial agents may act as a potent selective drive for promoting the emergence of resistant strains within the individual under antimicrobial use.

However, due to the transmissibility risk of infectious conditions [6,7], it is expected that the status of a certain subject affects the acquisition risk of this very same condition by others surrounding the original subject, a socalled ecological phenomenon. It would then be expected that this principle also applies to population exposures to antimicrobial drugs which, in turn, may lead to microor- 
ganisms' modifications with occasional resistance emergence. In other words, microorganisms exposed to antimicrobials within an individual or within a group of individuals (population) may be driven to modifications (resistance emergence); and modifications, once established, affect nearby neighbors by transmission (ecological effect). Due to ecological effect (or transmissibility potential), it is expected that population exposures to antimicrobial drugs may lead to microorganisms' modifications, occasionally leading to resistance emergence. The present review was based on previous empirical data and on related literature search for quantitative empirical models exploring the human-bacterial interactions.

\section{REVIEW}

We conducted previous experiments to understand the emergence of resistance in light of population selective forces [8,9]. A potential explanatory model was used to explore empirical observational data of human-microbiome interaction, i.e. on the one hand outpatient antimicrobial (ciprofloxacin and quinolone) consumption, as a global indicator of population antimicrobial exposure; and on the other hand community urinary tract (UTI) infections by E. coli, as an indicator of temporal and spatial pattern of bacterial resistance emergence. The underlying concept was that antibiotic use or consumption would act as a population trigger for pressing towards microbiome modifications. Thus, the hypothesis investigated was based on the rational that the emergence of bacterial resistance and possibly its dissemination would be related to spatial and temporal patterns of population antimicrobial consumption. Since there is vast documentation on E. coli quinolone resistance emergence related to individual antibiotic intake [10-12], quinolone consumption pattern was then used for this first study phase. The articles [8,9] have shown that the emergence of community urinary tract ciprofloxacin resistant (CIP-R). $E$. coli was significantly related to the previous three months levels of ciprofloxacin consumption between 5 and 9 Defined Daily Doses (DDD)/1000 inhabitants-day and to urban clusters of CIP-R E. coli in the city of São Paulo, Brazil, even after controlling for ciprofloxacin consumption. The generalized additive model (GAM) [13] was applied over one year observational sample of routine urine culture geo-coded results from two major outpatient centers showing 4372 E. coli urinary tract infections cases, with 723 ciprofloxacin resistant/intermediate cases. There was a clear evidence of hot-spots in the city associated to elevated risk of $E$. coli ciprofloxacin resistance acquisition. As investigated by the spatial analytical framework, significant spatial clustering of antimicrobial resistance was shown, even after controlling for population consumption, reinforcing the ecological effect hypothesis. The model $[8,9]$ has been applied on a longitudinal observational study, and as such, conclusions are limited to the environment used, even considering the elevated sample size and the goodness of fit of the model. As a cluster-randomized study of antimicrobial resistance, we applied the ratio of resistant to susceptible as an endpoint. The spatial clustering of antimicrobial resistance in this population pointed to an ecological effect, i.e., the results suggested that a human population submitted to a certain ciprofloxacin usage levels (5 to 9 DDD/1000 inhabitants-day) affected others around, not necessarily due to individual antimicrobial intake, but as a driving pressure over a modified circulating $E$. coli population (ciprofloxacin/quinolone resistance clusters). In other words, a certain population quinolone consumption pattern increased nearby neighbours' risk of resistant $E$. coli acquisition.

As previously noted [6,7], a population could experience indirect effects of antimicrobial use, such as an enhanced risk for resistance acquisition, because of antimicrobial usage by others in the population. Nevertheless, it is still unknown how these different environmental and individual determinants are distributed over space and time and their possible influences on a resistance emergence or clonal spread. It has been shown by time-series analysis that antimicrobial usage in a restricted and contained environment, such as a hospital, is temporally linked to the emergence of bacterial resistance $[14,15]$. Different epidemiological determinants may favour the emergence or establishment of specific resistances in given environments. Recently, the occurrence of an ecological phenomenon has been demonstrated [16] by a single clonal group accounting for nearly half of community-acquired urinary tract infections in women caused by E. coli with resistance to trimethoprim-sulfamethoxazole in three geographically diverse communities. In a different study, a geographical information system and a Bernoulli regression model were applied to detect clusters of higher risk of acquisition of $S$. aureus soft tissue abscesses [17]. Also, there have been strategies used with spatial scan statistics to identifying clusters of samples and to detecting areas with significantly high or low sampling rates of a national antimicrobial resistance monitoring program [18]. Jones et al. [19] applied a spatial analysis methodology and found more than half of emerging infectious diseases events to be caused by bacteria or rickettsia, with a large number of drug-resistance and significant correlation with socioeconomic, environmental and ecological factors. These approaches, including the present one, adopt a population-level perspective to elucidate the infection emergence problem and risk factors associated, in particular the resistance issue.

\section{DISCUSSIONS}

It has been shown that resistance lies within the human 
commensal bacterial species, which were once considered relatively harmless residents of the human microbiome [20]. It is still vastly unknown how different determinants are distributed over space and time and their possible influences on a microbiome, in particular on a specific epiphenomena such as clonal resistance emergence and spread. However, it seems reasonable to speculate that a human-driven increase in antibiotic concentrations of a given ecosystems, such as a city, may influence both antibiotic resistance and the microbial population dynamics [21]. Based on this line of thinking and on empirical findings [8,9,14-19], it is reasonably expected to observe a natural derivation of a bacterial population ontogeny under a certain level of exposure to external factors. This seems to be the case for the human commensal E. coli under ciprofloxacin/quinolone exposure, which would then derive to a resistant population and would occasionally be related to human infectious processes. This seems to be in accordance with the autopoietic theory $[22,23]$ and, thus, bacteria in general and $E$. coli in particular would be representatives of a secondary autopoietic structure, with their existence determined as a network part of a greater system [23]. As such, parts of this system if submitted to certain pressures could modify the whole system. Hence, as previously demonstrated [24], enterobacteria has an significant potential for adaptation, which in general could help explain how antibiotic resistance and virulence evolve so quickly, and in particular why the emergence of $E$. coli resistance was detected in geographical clusters and was related to population antimicrobial exposures (and not to individual ones) $[25,26]$.

\section{CONCLUSIONS}

Human populations submitted to a certain ciprofloxacin or quinolone usage level may affect neighbours within certain geographical areas, not necessarily due to individual antimicrobial intake, but as a driving pressure over a modified circulating $E$. coli population (as demonstrated by ciprofloxacin/quinolone resistance clusters).

It seems reasonable to expect a natural derivation of a bacterial population ontogeny under a certain level of exposure to external factors, which may be better studied within a population and spatial-temporal framework.

The adopted spatial-temporal framework accepts the inclusion of any number of potential variables, making it possible to fit in the model virtually any antimicrobialmicroorganism combination for the study of resistance emergence.

The spatial-temporal modelling approach embedded into a Geographical Information System analytical framework may help understanding human-bacterial interactions based on any of their epiphenomena (antimicrobial consumption, antimicrobial resistance, geno/phenotypic characteristics).

\section{ACKNOWLEDGEMENTS}

This research was supported by a FAPESP Research Grant: 2006/ 53922-9 (http://www.fapesp.br/english/).

\section{REFERENCES}

[1] Harbarth, S. and Samore, M.H. (2005) Antimicrobial resistance determinants and future control. Emerging Infectious Diseases, 11, 794-801. doi:10.3201/eid1106.050167

[2] World Health Organization (2001) Department of Communicable Disease Surveillance and Response. WHO Global Strategy for Containment of Antimicrobial Resistance (WHO/CDS/CSR/DRS/2001.2).

[3] Black, R.E., Morris, S.S. and Bryce, J. (2003) Where and why are 10 million children dying every year? Lancet, 361, 2226-2234. doi:10.1016/S0140-6736(03)13779-8

[4] Woolhouse, M.E.J. (2008) Epidemiology: Emerging diseases go global. Nature, 451, 898-899. doi:10.1038/451898a

[5] Infectious Disease Society of America (2008) Bad bugs, no drugs, as antibiotic discovery stagnates, a public health crisis brews.

http://www.medicalnewstoday.com/medicalnews.php?ne wsid=11084

[6] Lipsitch, M. and Samore, M.H. (2002) Antimicrobial use and antimicrobial resistance: A population perspective. Emerging Infectious Diseases, 8, 347-354. doi:10.3201/eid0804.010312

[7] Anderson, R.M. and May, R.M. (1979) Population boilogy of infectious diseases: Part I. Nature, 280, 361-367. doi:10.1038/280361a0

[8] Kiffer, C.R.V., Camargo, E.C.G., Shimakura, S.E., Ribeiro Jr., P.J., Bailey, T.C., Pignatari, A.C.C. and Monteiro, A.M.V. (2011) A spatial approach for the epidemicology of antibiotic use and resistance in community-based studies: The emergence of urban clusters of Escherichia coli quinolone resistance in Sao Paulo, Brasil. International Journal of Health Geographics, 10, 10-17. doi:10.1186/1476-072X-10-17

[9] Camargo, E.C.G., Kiffer, C.R.V., Pignatari, A.C.C., Shimakura, S.E., Ribeiro Jr., P.J. and Monteiro, A.M.V. (2012) Proposta sobre uso de dados de receitas de antimicrobianos retidas: A experiência EUREQA. Cad Saúde Pública, Rio de Janeiro, 28, 985-990. doi:10.1590/S0102-311X2012000500017

[10] Peña, C., Albareda, J.M., Pallares, R., Pujol, M., Tubau, F. and Ariza, J. (1995) Relationship between quinolone use and emergence of ciprofloxacin-resistant Escherichia coli in bloodstream infections. Antimicrobial Agents and Chemotherapy, 39, 520-524. doi:10.1128/AAC.39.2.520

[11] Yasufuku, T., Shigemura, K., Shirakawa, T., Matsumoto, M., Nakano, Y., Tanaka, K., Arakawa, S., Kinoshita, S., Kawabata, M. and Fujisawa, M. (2011) Correlation of overexpression of efflux pump genes with antibiotic resis- 
tance in Escherichia coli strains clinically isolated from urinary tract infection patients. Journal of Clinical Microbiology, 49, 189-194. doi:10.1128/JCM.00827-10

[12] Gallini, A., Degris, E., Desplas, M., Bourrel, R., Archambaud, M., Montastruc, J.-L., Lapeyre-Mestre, M. and Sommet, A. (2010) Influence of fluoroquinolone consumption in inpatients and outpatients on ciprofloxacin-resistant Escherichia coli in a university hospital. Journal of Antimicrobal Chemotherapy, 65, 2650-2657. doi:10.1093/jac/dkq351

[13] Wood, S. N. (2006) Generalized additive models-An introduction with $R$. Chapman and Hall, Boca Raton.

[14] Aldeyab, M.A., et al. (2008) Modelling the impact of antibiotic use and infection control practices on the incidence of hospital-acquired methicillin-resistant staphylococcus aureus: A time-series analysis. Journal of Antimicrobial Chemotherapy, 62, 593-600. doi:10.1093/jac/dkn198

[15] López-Lozano, J.M., et al. (2000) Modelling and forecasting antimicrobial resistance and its dynamic relationship to antimicrobial use: A time series analysis. International Journal of Antimicrobial Agents, 14, 21-31. doi:10.1016/S0924-8579(99)00135-1

[16] Manges, A.R., Johnson, J.R., Foxman, B., O’Bryan, T.T., Fullerton, K.E., et al. (2001) Widespread distribution of urinary tract infections caused by a multidrug-resistant Escherichia coli clonal group. New England Journal of Medicine, 345, 1007-1013. doi:10.1056/NEJMoa011265

[17] Tirabassi, M.V., Wadie, G., Moriarty, K.P., Garb, J., Konefal, S.H., et al. (2005) Geographic information system localization of community-acquired MRSA soft tissue abscesses. Journal of Pediatric Surgery, 40, 962-966. doi:10.1016/j.jpedsurg.2005.03.010

[18] Vieira, A.R., Houe, H., Wegener, H.C., Wong Lo Fo, D.M.A., Bodker, R., et al. (2009) Spatial scan statistics to assess sampling strategy of antimicrobial resistance mo- nitoring program. Foodborne Pathogens and Disease, 6, 15-21. doi:10.1089/fpd.2008.0132

[19] Jones, K.E., Patel, N.G., Levy, M.A., Storeygard, A., Balk, D., et al. (2008) Global trends in emerging infectious diseases. Nature, 451, 990-994. doi:10.1038/nature06536

[20] Morten, O.A.S., Dantas, G. and Church, G.M. (2009) Functional Characterization of the Antibiotic Resistance Reservoir in the Human Microflora. Science, 325, 1128. doi:10.1126/science.1176950

[21] Martinez, J.L. (2008) Antibiotics and antibiotic resistance genes in natural environments. Science, 321, 365-367. doi:10.1126/science.1159483

[22] Varela, F.J., Maturana, H.R. and Uribe, R. (1974) Autopoiesis: The organization of living systems, its characterization and a model. Biosystems, 5, 187. doi:10.1016/0303-2647(74)90031-8

[23] Fleischaker, G.R. (1988) Autopoiesis: The status of its system logic. Biosystems, 22, 37-49. doi:10.1016/0303-2647(88)90048-2

[24] Perfeito, L., Fernandes, L., Mota, C. and Gordo, I. (2007) Adaptive mutations in bacteria: High Rate and small effects. Science, 317, 813-815. doi:10.1126/science.1142284

[25] Linares, J.F., Gustafsson, I., Baquero, F. and Martinez, J.L. (2006) Antibiotics as intermicrobial signaling agents instead of weapons. PNAS, 103, 19484-19489. doi:10.1073/pnas.0608949103

[26] Pallecchi, L., Riccobono, E., Mantella, A., Fernandez, C., Bartalesi, F., Rodriguez, H., Gotuzzo, E., Bartoloni, A. and Rossolini, G.N. (2011) Small qnrB-harbouring ColElike plasmids widespread in commensal enterobacteria from a remote Amazonas population not exposed to antibiotics. Journal of Antimicrobial Chemotherapy, 66, 1176-1178. doi:10.1093/jac/dkr026 